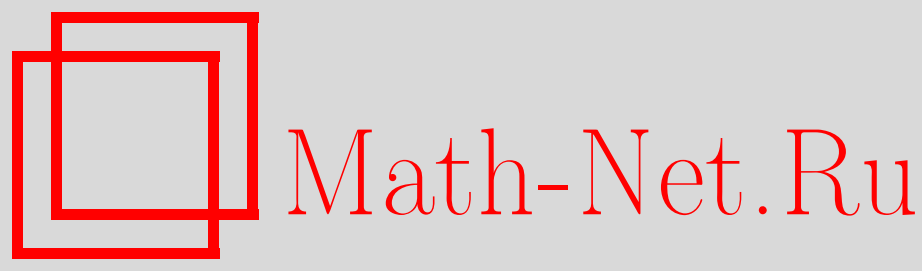

А. А. Андрианов, А. В. Соколов, Расширенная суперсимметрия и скрытые симметрии в одномерной матричной квантовой механике, ТМФ, 2016, том 186, номер 1, 5-26

DOI: https://doi.org/10.4213/tmf8977

Использование Общероссийского математического портала Math-Net.Ru подразумевает, что вы прочитали и согласны с пользовательским соглашением http://www.mathnet.ru/rus/agreement

Параметры загрузки:

IP : 54.81 .137 .203

26 апреля 2023 г., $11: 47: 49$

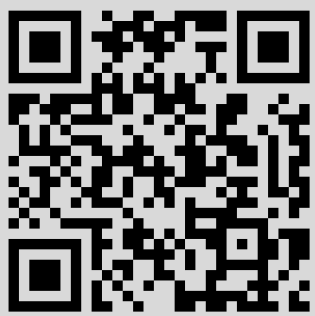




\title{
ФИЗИКА
}

Том 186, № 1

январь, 2016

\section{РАСШИРЕННАЯ СУПЕРСИММЕТРИЯ И СКРЫТЫЕ СИММЕТРИИ В ОДНОМЕРНОЙ МАТРИЧНОЙ КВАНТОВОЙ МЕХАНИКЕ}

\begin{abstract}
Исследованы свойства нелинейных алгебр суперсимметрии, реализующиеся в одномерной квантовой механике матричных систем. Суперзаряды этих алгебр являются дифференциальными операторами конечного порядка по производным. В особых случаях существуют несколько независимых суперзарядов, которые осуществляют (расширенную) суперсимметрию одного и того же супергамильтониана. Расширенная суперсимметрия порождает скрытые симметрии супергамильтониана. Такие симметрии найдены в моделях с $(2 \times 2)$-матричными потенциалами.
\end{abstract}

Ключевые слова: матричный гамильтониан, расширенная алгебра суперсимметрии, скрытая симметрия.

DOI: $10.4213 / \operatorname{tmf} 8977$

\section{1. ВВЕДЕНИЕ}

Метод суперсимметрии в квантовой механике служит для построения изоспектральных или почти изоспектральных квантовых гамильтонианов с различными потенциалами (см. обзоры [1]-[4]). Наиболее развитая схема построения изоспектральных систем основана на нелинейной (полиномиальной) алгебре суперсимметрии (см. обзор [4]). Она была детально разработана в последнее десятилетие для скалярных потенциалов [5]-[15].

Работа выполнена при поддержке РФФИ (гранты № 13-01-00136_а, № 16-0100375 _а). Авторы признательны Санкт-Петербургскому государственному университету за исследовательский грант № 11.38.660.2013 и за гранты № 11.41.847.2015 (А. В. Соколов) и № 11.41.775.2015 (А. А. Андрианов) для участия в конференции PHHQP'15.

* Санкт-Петербургский государственный университет, Санкт-Петербург, Россия. E-mail: a.andrianov@spbu.ru, andrey.sokolov@spbu.ru 
Вместе с тем заслуживает серьезного внимания спектральный дизайн для матричных потенциалов, так как целый ряд задач многоканального и многочастичного рассеяния, а также задач о взаимодействии частиц со спином содержат матричные потенциалы, управляющие квантовой динамикой. В этой области сделал вклад и Станислав Петрович Меркурьев: он использовал методы суперсимметричной механики для получения многочастичных потенциалов без нефизических уровней энергии [16].

Суперсимметричная механика избранных матричных систем имеет довольно солидную историю [17]-[45]. Однако систематические шаги в развитие спектрального дизайна для матричных потенциалов были сделаны лишь в последние три года [41], [46], [47]. В частности, найдены простые формулы, позволяющие построить любой $(n \times n)$-матричный сплетающий оператор произвольного порядка и соответствующий новый гамильтониан в терминах формальных собственных и присоединенных вектор-функций исходного гамильтониана. Обнаружено, что для любого матричного сплетающего оператора наименьшего порядка существует матричный оператор, сплетающий те же гамильтонианы в противоположном направлении, с помощью которого можно построить полиномиальную алгебру суперсимметрии. Найдены критерии приводимости и слабой, и сильной минимизуемости суперсимметричных алгебр, а также показано, что существуют абсолютно неприводимые матричные сплетающие операторы. Исследованы условия существования матрицы симметрии для матричного гамильтониана и с их помощью разработан удобный метод построения матричных гамильтонианов с заданной матрицей симметрии.

Настоящая работа посвящена более детальному определению нелинейных алгебр суперсимметрии для матричных потенциалов в одномерной квантовой механике, которое содержится в теореме 1. Суперзаряды этих алгебр являются дифференциальными операторами конечного порядка по производным. В некоторых случаях существуют несколько независимых суперзарядов, которые осуществляют (расширенную) суперсимметрию одного и того же супергамильтониана. Расширенная суперсимметрия порождает скрытые симметрии супергамильтониана [8], [48]. Такие симметрии найдены в различных моделях с $(2 \times 2)$-матричными потенциалами (см. также примеры в [26]). В этих моделях построены примеры скрытых симметрий, которые порождают линейные и нелинейные алгебры суперсимметрии в квантовой механике матричных систем, и проведен анализ алгоритмов их поиска.

\section{2. ОСНОВНЫЕ ОПРЕДЕЛЕНИЯ И ОБОЗНАЧЕНИЯ}

2.1. Сплетающий оператор. Рассмотрим два определенных на всей оси матричных гамильтониана шредингеровского вида

$$
H_{+}=-I_{n} \partial^{2}+V_{+}(x), \quad H_{-}=-I_{n} \partial^{2}+V_{-}(x), \quad \partial \equiv \frac{d}{d x},
$$

где $I_{n}-$ единичная матрица $n$-го порядка, $n \in \mathbb{N}$, и $V_{+}(x)$ и $V_{-}(x)-(n \times n)$-матричные потенциалы, все элементы которых представляют собой достаточно гладкие функции, принимающие, вообе говоря, комплексные значения. Будем предполагать, что эти гамильтонианы сплетаются $(n \times n)$-матричным линейным дифференциальным оператором $N$-го порядка $Q_{N}^{-}$(сплетающим оператором $), N \in \mathbb{N} \cup\{0\}$, так, 
что

$$
Q_{N}^{-} H_{+}=H_{-} Q_{N}^{-}, \quad Q_{N}^{-}=\sum_{j=0}^{N} X_{j}^{-}(x) \partial^{j},
$$

и все элементы $(n \times n)$-матричных коэффициентов $X_{j}^{-}(x), j=0,1, \ldots, N$, этого оператора также являются достаточно гладкими и принимают, вообе говоря, комплексные значения.

Из (2) следует [41], что старший коэффициент $X_{N}^{-}(x)$ представляет собой постоянную матрицу и

$$
V_{-}(x)=X_{N}^{-} V_{+}(x)\left(X_{N}^{-}\right)^{-1}+2 X_{N-1}^{-\prime}(x)\left(X_{N}^{-}\right)^{-1}, \quad \text { если } \quad \operatorname{det} X_{N}^{-} \neq 0 .
$$

Будем в дальнейшем предполагать, что $\operatorname{det} X_{N}^{-} \neq 0$.

2.2. Структура ядра сплетающего оператора. Поскольку ядро оператора $Q_{N}^{-}$в силу (2) является инвариантным относительно $H_{+}$,

$$
H_{+} \operatorname{ker} Q_{N}^{-} \subset \operatorname{ker} Q_{N}^{-}
$$

постольку для любого базиса $\Phi_{1}^{-}(x), \ldots, \Phi_{d}^{-}(x), d=\operatorname{dim} \operatorname{ker} Q_{N}^{-}=n N$, в этом ядре существует постоянная квадратная $(d \times d)$-матрица $\mathbf{T}^{+} \equiv\left\|T_{i j}^{+}\right\|$, для которой справедливы следующие равенства:

$$
H_{+} \Phi_{i}^{-}=\sum_{j=1}^{d} T_{i j}^{+} \Phi_{j}^{-}, \quad i=1,2, \ldots, d .
$$

При этом вронскиан всех элементов любого базиса в ядре $Q_{N}^{-}$не имеет корней.

Матрищей $\mathbf{T}$ сплетающего оператора будем называть матрицу, которая строится для этого оператора таким же образом, каким матрица $\mathbf{T}^{+}$была построена для оператора $Q_{N}^{-}$. При этом базис в ядре сплетающего оператора, как правило, не будет указываться в тех случаях, когда рассматриваются только спектральные характеристики матрицы $\mathbf{T}$, или, что то же самое, спектральные характеристики сужения соответствующего гамильтониана на ядро рассматриваемого сплетающего оператора (ср. с (4)).

Базис в ядре сплетающего оператора, который обеспечивает нормальный (жорданов) вид матрице $\mathbf{T}$ этого оператора, будем называть каноническим базисом. Элементы канонического базиса называются преобразующими вектор-функииями.

В тех случаях, когда нормальная форма матрицы $\mathbf{T}$ сплетающего оператора содержит клетку большего порядка (клетки больших порядков), чем первый, соответствующий канонический базис включает в себя кроме формальной собственной вектор-функции (формальных собственных вектор-функций) соответствующего гамильтониана также и его формальную присоединенную вектор-функцию (формальные присоединенные вектор-функции), которая определяется (которые определяются) следующим образом [49]. 
Вектор-функция $\Phi_{m, i}(x)$ называется формальной присоединенной вектор-функицей $i$-го порядка $(n \times n)$-матричного гамильтониана $H=-I_{n} \partial^{2}+V(x)$ для спектрального числа $\lambda_{m}$, если

$$
\left(H-\lambda_{m} I_{n}\right)^{i+1} \Phi_{m, i} \equiv 0, \quad\left(H-\lambda_{m} I_{n}\right)^{i} \Phi_{m, i} \not \equiv 0 .
$$

При этом слово “формальный” подчеркивает, что вектор-функция $\Phi_{m, i}(x)$ не обязательно принадлежит $L^{2}\left(\mathbb{R}, \mathbb{C}^{n}\right)$ (не обязательно нормируема). Формальную присоединенную вектор-функцию нулевого порядка $\Phi_{m, 0}(x)$ в этом случае естественно назвать формальной собственной вектор-функиией гамильтониана $H$.

Конечный или бесконечный набор вектор-функций $\Phi_{m, i}(x), i=0,1,2, \ldots$, называется цепочкой формальных присоединенных вектор-функций $(n \times n)$-матричного гамильтониана $H=-I_{n} \partial^{2}+V(x)$ для спектрального числа $\lambda_{m}$, если

$$
H \Phi_{m, 0}=\lambda_{m} \Phi_{m, 0}, \quad \Phi_{m, 0}(x) \not \equiv 0, \quad\left(H-\lambda_{m} I_{n}\right) \Phi_{m, i}=\Phi_{m, i-1}, \quad i=1,2, \ldots
$$

Очевидно, что $\Phi_{m, i}(x)$ при этом является формальной присоединенной вектор-функцией $i$-го порядка гамильтониана $H$ для спектрального числа $\lambda_{m}, i=0,1,2, \ldots$.

2.3. Слабая и сильная минимизуемость сплетающего оператора. Если оператор $Q_{N}^{-}$умножить на многочлен от гамильтониана:

$$
Q_{N}^{-}\left[\sum_{l=0}^{L} A_{l} H_{+}^{l}\right] \quad\left(\left[\sum_{l=0}^{L} A_{l} H_{-}^{l}\right] Q_{N}^{-}\right)
$$

где коэффициенты $A_{l}, l=0,1, \ldots, L$, являются либо комплексными числами, либо постоянными матрицами симметрии для гамильтониана $H_{+}\left(H_{-}\right)$, то возникшее произведение, очевидно, снова будет сплетающим оператором для той же самой пары гамильтонианов:

$$
\begin{gathered}
\left\{Q_{N}^{-}\left[\sum_{l=0}^{L} A_{l} H_{+}^{l}\right]\right\} H_{+}=Q_{N}^{-} H_{+}\left[\sum_{l=0}^{L} A_{l} H_{+}^{l}\right]=H_{-}\left\{Q_{N}^{-}\left[\sum_{l=0}^{L} A_{l} H_{+}^{l}\right]\right\} \\
\left(\left\{\left[\sum_{l=0}^{L} A_{l} H_{-}^{l}\right] Q_{N}^{-}\right\} H_{+}=\left[\sum_{l=0}^{L} A_{l} H_{-}^{l}\right] H_{-} Q_{N}^{-}=H_{-}\left\{\left[\sum_{l=0}^{L} A_{l} H_{-}^{l}\right] Q_{N}^{-}\right\}\right) .
\end{gathered}
$$

Такие полиномиальные сомножители во многих случаях могут содержаться в рассматриваемых сплетающих операторах и неоправданно усложнять соответствующие алгебры. Для рассмотрения возможности отщепления от заданного сплетающего оператора излишнего полиномиального сомножителя удобно ввести понятия слабой и сильной минимизуемости [41], [47], совпадающие в скалярном случае $n=1$ и переходящие в этом случае в соответствующее понятие, введенное в [8], [12]. В настоящей статье мы ограничимся рассмотрением только слабой минимизуемости сплетающего оператора. 
Будем называть сплетающий оператор $Q_{N}^{-}$слабо минимизуемъмм, если он может быть представлен в виде следующих произведений:

$$
\begin{gathered}
Q_{N}^{-}=P_{M}^{-}\left[\sum_{l=0}^{L} a_{l} H_{+}^{l}\right] \equiv\left[\sum_{l=0}^{L} a_{l} H_{-}^{l}\right] P_{M}^{-}, \\
a_{l} \in \mathbb{C}, \quad l=0,1, \ldots, L, \quad a_{L} \neq 0, \quad 1 \leqslant L \leqslant N / 2,
\end{gathered}
$$

где $P_{M}^{-}-(n \times n)$-матричный линейный дифференциальный оператор $M$-го порядка, $M=N-2 L$, который сплетает гамильтонианы $H_{+}$и $H_{-}$так, что $P_{M}^{-} H_{+}=H_{-} P_{M}^{-}$. В противном случае оператор $Q_{N}^{-}$будем называть слабо неминимизуемым.

\section{3. ПОЛИНОМИАЛЬНАЯ АЛГЕБРА СУПЕРСИММЕТРИИ ДЛЯ ПРОИЗВОЛЬНОГО СПЛЕТАЮЩЕГО ОПЕРАТОРА}

Существование полиномиальной алгебры суперсимметрии для любого матричного сплетающего оператора с невырожденным матричным коэффициентом при операторе дифференцирования в старшей степени является следствием ряда требований, которые мы сформулируем в условиях приводимой ниже теоремы.

ТЕОРЕма 1. Пусть выполняются следующие условия:

1) $(n \times n)$-матричные гамильтонианы $H_{+} u H_{-}$вида (1) сплетаются $(n \times n)$ матричным линейным дифференциальным оператором $N$-го порядка $Q_{N}^{-}$вида (2) с постоянным невырожденным матричным коэббиииентом $X_{N}^{-} n p и \partial^{N}$ в соответствии с (2);

2) $T^{+}$- матрица $T$ сплетающего оператора $Q_{N}^{-}$;

3) $\lambda_{l}, l=1,2, \ldots, L,-$ полный набор различных собственных чисел матрицъ $T^{+}$;

4) $g_{l}^{-}$- геометрическая кратность собственного числа $\lambda_{l}$ матрищъ $T^{+}, l=$ $1,2, \ldots, L$

5) $k_{l, j}^{-}, j=1,2, \ldots, g_{l}^{-},-$порядки жсордановых клеток в нормальной форме матрицы $T^{+}$, которые соответствуют собственному числу $\lambda_{l}, l=1,2, \ldots, L$, причем последовательность $k_{l, j}^{-}, j=1,2, \ldots, g_{l}^{-}$, при любом $l=1,2, \ldots, L$ является невозрастающей;

6) $\varkappa_{l}=\max _{1 \leqslant j \leqslant g_{l}^{-}} k_{l, j}^{-}, l=1,2, \ldots, L$.

Тогда справедливы следующие утверждения:

1) существует единственный слабо неминимизуемый $(n \times n)$-матричный линейный дифференииалъный оператор $Q_{N^{\prime}}^{+}$, который сплетает $H_{+} u H_{-}$так, что

$$
H_{+} Q_{N^{\prime}}^{+}=Q_{N^{\prime}}^{+} H_{-},
$$

и такой, что произведение $Q_{N^{\prime}}^{+} Q_{N}^{-}$представляет собой многочлен с числовыми коэффициентами от гамильтониана $H_{+}$и единичным коэффициентом при $H_{+}$ в старшей степени;

2) существует единственный слабо неминимизуемый $(n \times n)$-матричный линейный дифференциальный оператор $\hat{Q}_{N^{\prime}}^{+}$, который сплетает $H_{+}$u $H_{-}$maк, что

$$
H_{+} \hat{Q}_{N^{\prime}}^{+}=\hat{Q}_{N^{\prime}}^{+} H_{-},
$$


и такой, что произведение $Q_{N}^{-} \hat{Q}_{N^{\prime}}^{+}$представляет собой многочлен с числовыми коэффициентами от гамильтониана $H_{-}$и единичным коэффициентом при $H_{-}$ в старшей степени;

3) $Q_{N^{\prime}}^{+}=\hat{Q}_{N^{\prime}}^{+}$;

4) порядок $N^{\prime}$ оператора $Q_{N^{\prime}}^{+}=\hat{Q}_{N^{\prime}}^{+}$можно найти по формуле

$$
N^{\prime}=-N+2 \sum_{l=1}^{L} \varkappa_{l}
$$

5) справедливы равенства

$$
\begin{gathered}
Q_{N^{\prime}}^{+} Q_{N}^{-}=\mathcal{P}_{\left(N+N^{\prime}\right) / 2}\left(H_{+}\right), \quad Q_{N}^{-} Q_{N^{\prime}}^{+}=\mathcal{P}_{\left(N+N^{\prime}\right) / 2}\left(H_{-}\right), \\
\mathcal{P}_{\left(N+N^{\prime}\right) / 2}(\lambda) \equiv \prod_{l=1}^{L}\left(\lambda-\lambda_{l}\right)^{\varkappa_{l}}
\end{gathered}
$$

Доказательство теоремы 1 занимает несколько страниц журнального текста и будет опубликовано в другой статье. Здесь мы ограничимся ее применением к конкретным $(2 \times 2)$-матричным гамильтонианам.

СЛЕДСТВИЕ 1. Для любого $(n \times n)$-матричного сплетающего оператора $Q_{N}^{-}$ с невырожденным матричным коэфбициентом при $\partial^{N}$, используя супергамильтониан

$$
\mathbf{H}=\left(\begin{array}{cc}
H_{+} & 0 \\
0 & H_{-}
\end{array}\right)
$$

и суперзарядъ

$$
\mathbf{Q}=\left(\begin{array}{cc}
0 & Q_{N}^{+} \\
0 & 0
\end{array}\right), \quad \overline{\mathbf{Q}}=\left(\begin{array}{cc}
0 & 0 \\
Q_{N}^{-} & 0
\end{array}\right), \quad \mathbf{Q}^{2}=\overline{\mathbf{Q}}^{2}=0
$$

можно построить следующую полиномиалъную алгебру суперсимметрии:

$$
\{\mathbf{Q}, \overline{\mathbf{Q}}\}=\mathcal{P}_{\left(N+N^{\prime}\right) / 2}(\mathbf{H}), \quad[\mathbf{H}, \mathbf{Q}]=[\mathbf{H}, \overline{\mathbf{Q}}]=0
$$

\section{4. ПОИСК $(2 \times 2)$-МАТРИЧНЫХ ОПЕРАТОРОВ СИММЕТРИИ}

Рассмотрим модель, в которой два симметричных $(2 \times 2)$-матричных гамильтониана $H_{+}$и $H_{-}$сплетаются операторами первого и нулевого порядков:

$$
\begin{gathered}
Q_{1}^{-} H_{+}=H_{-} Q_{1}^{-}, \quad Q_{1}^{-}=X_{1}^{-} \partial+X_{0}^{-}, \quad H_{ \pm}^{\mathrm{t}}=H_{ \pm}, \\
P_{0}^{-} H_{+}=H_{-} P_{0}^{-}, \quad P_{0}^{-}=Y_{0}^{-}, \quad \operatorname{det} Y_{0}^{-}=1 .
\end{gathered}
$$

Принимая во внимание симметричность гамильтонианов $H_{+}$и $H_{-}$, нетрудно увидеть, что операторы $\left(Q_{1}^{-}\right)^{\mathrm{t}}$ и $\left(P_{0}^{-}\right)^{\mathrm{t}}$ сплетают эти гамильтонианы в противоположном направлении:

$$
\left(Q_{1}^{-}\right)^{\mathrm{t}} H_{-}=H_{+}\left(Q_{1}^{-}\right)^{\mathrm{t}}, \quad\left(P_{0}^{-}\right)^{\mathrm{t}} H_{-}=H_{+}\left(P_{0}^{-}\right)^{\mathrm{t}} .
$$


Из операторов $Q_{1}^{-}, P_{0}^{-},\left(Q_{1}^{-}\right)^{\mathrm{t}}$ и $\left(P_{0}^{-}\right)^{\mathrm{t}}$ для $H_{+}$и $H_{-}$, очевидно, можно составить операторы симметрии, антисимметричные и симметричные относительно транспонирования:

$$
\begin{array}{lll}
R_{\mathrm{a}, \mathrm{s}}^{+}=\left(Q_{1}^{-}\right)^{\mathrm{t}} P_{0}^{-} \mp\left(P_{0}^{-}\right)^{\mathrm{t}} Q_{1}^{-}, & \left(R_{\mathrm{a}, \mathrm{s}}^{+}\right)^{\mathrm{t}}=\mp R_{\mathrm{a}, \mathrm{s}}^{+}, & {\left[R_{\mathrm{a}}, H_{+}\right]=\left[R_{s}, H_{+}\right]=0,} \\
R_{\mathrm{a}, \mathrm{s}}^{-}=Q_{1}^{-}\left(P_{0}^{-}\right)^{\mathrm{t}} \mp P_{0}^{-}\left(Q_{1}^{-}\right)^{\mathrm{t}}, & \left(R_{\mathrm{a}, \mathrm{s}}^{+}\right)^{\mathrm{t}}=\mp R_{\mathrm{a}, \mathrm{s}}^{+}, & {\left[R_{\mathrm{a}}, H_{+}\right]=\left[R_{s}, H_{+}\right]=0 .}
\end{array}
$$

Ограничимся исследованием только операторов $R_{\mathrm{a}}^{+}$и $R_{\mathrm{s}}^{+}$, поскольку операторы $R_{\mathrm{a}}^{-}$ и $R_{\mathrm{s}}^{-}$могут быть исследованы таким же образом. Кроме того, отметим, что в силу (9) потенциалы гамильтонианов $H_{+}$и $H_{-}$являются подобными:

$$
H_{-}=Y_{0}^{-} H_{+}\left(Y_{0}^{-}\right)^{-1} \quad \Longleftrightarrow \quad V_{-}(x)=Y_{0}^{-} V_{+}(x)\left(Y_{0}^{-}\right)^{-1}
$$

Последнее равенство совместно с равенством

$$
Q_{1}^{-}=\frac{1}{2}\left(\left(Y_{0}^{-}\right)^{\mathrm{t}}\right)^{-1}\left(R_{\mathrm{s}}^{+}-R_{\mathrm{a}}^{+}\right)
$$

следующим из $(9)$ и $(10)$, оно позволяет выразить гамильтониан $H_{-}$и сплетающий оператор $Q_{1}^{-}$через $H_{+}, R_{\mathrm{a}}^{+}, R_{\mathrm{s}}^{+}$и $P_{0}^{-}=Y_{0}^{-}$либо, если предположить, что изначально известны только $H_{+}=H_{+}^{\mathrm{t}}, R_{\mathrm{a}}^{+}$и $R_{\mathrm{s}}^{-}$, найти $(2 \times 2)$-матричный гамильтониан $H_{-}$, сплетаемый с $H_{+}$оператором $P_{0}^{-}=Y_{0}^{-}$и оператором (11) так, что имеют место соотношения (9). При этом нетрудно проверить, что для того, чтобы гамильтониан $H_{-}$ был симметричным, необходимо и достаточно, чтобы элементы матрицы $Y_{0}^{-} \equiv\left\|y_{i j}^{-}\right\|$ удовлетворяли соотношению

$$
\begin{gathered}
\left(y_{11}^{-} y_{12}^{-}+y_{21}^{-} y_{22}^{-}\right)\left(v_{11}^{+}(x)-v_{22}^{+}(x)\right)+\left(\left(y_{12}^{2}+y_{22}^{2}\right)-\left(y_{11}^{2}+y_{21}^{2}\right)\right) v_{12}^{+}(x) \equiv 0, \\
V_{+}(x) \equiv\left\|v_{i j}^{+}(x)\right\|,
\end{gathered}
$$

которое будет справедливо, если, например, выбрать матрицу $Y_{0}^{-}$ортогональной.

\section{1. Антисимметричный оператор симметрии с диагонализуемым стар-} шим коэффициентом. У антисимметричного оператора симметрии $R_{\mathrm{a}}^{+}$коэффициент при $\partial$ представляет собой симметричную матрицу. Предположим, что существует ортогональная матрица $A$, приводящая этот коэффициент к диагональному виду. Антисимметричный матричный коэффициент $R_{\mathrm{a}}^{+}$при $\partial^{0}$ при указанном преобразовании, очевидно, останется антисимметричным, а симметричный гамильтониан $H_{+}$перейдет в некоторый новый симметричный гамильтониан. Таким образом, операторы $R_{\mathrm{a}}^{+}$и $H_{+}$можно представить в виде

$$
\begin{aligned}
& R_{\mathrm{a}}^{+}=A\left[\left(\begin{array}{cc}
\mu_{1} & 0 \\
0 & \mu_{2}
\end{array}\right) \partial+r_{0}(x)\left(\begin{array}{cc}
0 & 1 \\
-1 & 0
\end{array}\right)\right] A^{\mathrm{t}}, \\
& H_{+}=A\left[-\partial^{2} I_{2}+\left(\begin{array}{cc}
v_{1}(x)+v_{2}(x) & v_{3}(x) \\
v_{3}(x) & v_{1}(x)-v_{2}(x)
\end{array}\right)\right] A^{\mathrm{t}},
\end{aligned}
$$


где $\mu_{1}, \mu_{2}, r_{0}(x), v_{1}(x), v_{2}(x)$ и $v_{3}(x)$ - некоторые постоянные и функции, удовлетворяющие следующей системе уравнений:

$$
\begin{aligned}
\left(\mu_{1}-\mu_{2}\right) v_{3}(x)+2 r_{0}^{\prime}(x) & =0, \\
\mu_{1}\left(v_{1}^{\prime}(x)+v_{2}^{\prime}(x)\right)+2 r_{0}(x) v_{3}(x) & =0, \\
\mu_{2}\left(v_{1}^{\prime}(x)-v_{2}^{\prime}(x)\right)-2 r_{0}(x) v_{3}(x) & =0, \\
\mu_{1} v_{3}^{\prime}(x)-2 r_{0}(x) v_{2}(x)+r_{0}^{\prime \prime}(x) & =0, \\
\mu_{2} v_{3}^{\prime}(x)-2 r_{0}(x) v_{2}(x)-r_{0}^{\prime \prime}(x) & =0,
\end{aligned}
$$

равносильной условию перестановочности $R_{\mathrm{a}}^{+}$и $H_{+}$. Дальнейшее исследование проведем по отдельности для нескольких случаев.

4.1.1. Случай $\mu_{1} \mu_{2} \neq 0, \mu_{1}^{2} \neq \mu_{2}^{2}$. Покажем, что в этом случае $v_{1}(x), v_{2}(x)$ и $v_{3}(x)$ можно выразить через $r_{0}(x)$, и найдем уравнение, которому удовлетворяет $r_{0}(x)$. Умножив второе уравнение системы (12) на $\mu_{2}$, первое - на $\mu_{1}$, сложив результаты, подставив в полученное уравнение выражение для $\left(\mu_{1}-\mu_{2}\right) v_{3}(x)$ из первого уравнения и проинтегрировав, приходим к соотношению

$$
\mu_{1} \mu_{2} v_{1}(x)+r_{0}^{2}(x)=C_{1} \quad \Rightarrow \quad v_{1}(x)=\frac{C_{1}-r_{0}^{2}(x)}{\mu_{1} \mu_{2}},
$$

где $C_{1}$ - постоянная интегрирования. Вычитая из второго уравнения системы (12), умноженного на $\mu_{2}$, третье уравнение, умноженное на $\mu_{1}$, умножив результат на $\mu_{1}-\mu_{2}$, подставив в полученное уравнение выражение для $\left(\mu_{1}-\mu_{2}\right) v_{3}(x)$ из первого уравнения и проинтегрировав, получаем соотношение

$$
\mu_{1} \mu_{2}\left(\mu_{1}-\mu_{2}\right) v_{2}(x)-\left(\mu_{1}+\mu_{2}\right) r_{0}^{2}(x)=\left(\mu_{1}+\mu_{2}\right) C_{2},
$$

где $C_{2}$ - постоянная интегрирования. Вычитая из четвертого уравнения системы (12), умноженного на $\mu_{2}$, пятое уравнение, умноженное на $\mu_{1}$, получаем

$$
2\left(\mu_{1}-\mu_{2}\right) r_{0}(x) v_{2}(x)+\left(\mu_{1}+\mu_{2}\right) r_{0}^{\prime \prime}(x)=0 .
$$

Из (13) и (14) находим, что $v_{2}(x)$ можно выразить через $r_{0}(x)$ двумя способами:

$$
v_{2}(x)=\frac{\mu_{1}+\mu_{2}}{\mu_{1}-\mu_{2}} \frac{C_{2}+r_{0}^{2}(x)}{\mu_{1} \mu_{2}}=-\frac{1}{2} \frac{\mu_{1}+\mu_{2}}{\mu_{1}-\mu_{2}} \frac{r_{0}^{\prime \prime}(x)}{r_{0}(x)},
$$

и, таким образом, $r_{0}(x)$ удовлетворяет уравнению

$$
-\frac{1}{2} \mu_{1} \mu_{2} r_{0}^{\prime \prime}(x)=r_{0}^{3}(x)+C_{2} r_{0}(x)
$$

проинтегрировав которое, получаем

$$
\left(r_{0}^{\prime}\right)^{2}=-\frac{1}{\mu_{1} \mu_{2}}\left[r_{0}^{4}(x)+2 C_{2} r_{0}^{2}(x)+C_{3}\right],
$$


где $C_{3}$ - постоянная интегрирования. Наконец, из первого уравнения системы (12) следует, что

$$
v_{3}(x)=-\frac{2 r_{0}^{\prime}(x)}{\mu_{1}-\mu_{2}} .
$$

Вид общего решения уравнения (15), а тем самым и вид функций $v_{1}(x), v_{2}(x)$ и $v_{3}(x)$ определяются соотношением коэффициентов $C_{2}$ и $C_{3}$. Поэтому рассмотрим несколько частных случаев.

ЧАСТныЙ СЛУЧАЙ $C_{2}=C_{3}=0, r_{0}(x) \neq$ const. В этом случае общее решение уравнения (15) имеет вид

$$
r_{0}(x)=i \frac{\sqrt{\mu_{1}} \sqrt{\mu_{2}}}{x-x_{0}}, \quad \operatorname{Im} x_{0} \neq 0
$$

где $x_{0}$ - постоянная интегрирования, условие $\operatorname{Im} x_{0} \neq 0$ обеспечивает отсутствие вещественных полюсов, и ветви корней $\sqrt{\mu_{1}}$ и $\sqrt{\mu_{2}}$ могут выбираться произвольно. Соответственно оператор симметрии $R_{\mathrm{a}}^{+}$и гамильтониан $H_{+}$принимают вид

$$
\begin{aligned}
& R_{\mathrm{a}}^{+}=\sqrt{\mu_{1}} \sqrt{\mu_{2}} A\left[\left(\begin{array}{cc}
\frac{\sqrt{\mu_{1}}}{\sqrt{\mu_{2}}} & 0 \\
0 & \frac{\sqrt{\mu_{2}}}{\sqrt{\mu_{1}}}
\end{array}\right) \partial+\frac{i}{x-x_{0}}\left(\begin{array}{cc}
0 & 1 \\
-1 & 0
\end{array}\right)\right] A^{\mathrm{t}}, \\
& H_{+}=A\left[-I_{2} \partial^{2}+\frac{2}{\left(x-x_{0}\right)^{2}}\left(\begin{array}{cc}
-\frac{\mu_{2}}{\mu_{1}-\mu_{2}} & i \frac{\sqrt{\mu_{1}} \sqrt{\mu_{2}}}{\mu_{1}-\mu_{2}} \\
i \frac{\sqrt{\mu_{1}} \sqrt{\mu_{2}}}{\mu_{1}-\mu_{2}} & \frac{\mu_{1}}{\mu_{1}-\mu_{2}}
\end{array}\right)+\lambda_{0} I_{2}\right] A^{\mathrm{t},} \quad \lambda_{0}=\frac{C_{1}}{\mu_{1} \mu_{2}} .
\end{aligned}
$$

Используя ортогональную матрицу $B$, оператор $H_{+}$можно привести к более простому виду:

$R_{\mathrm{a}}^{+}=\sqrt{\mu_{1}} \sqrt{\mu_{2}} A B\left[\left(\begin{array}{cc}0 & -i \\ -i & \frac{\mu_{1}+\mu_{2}}{\sqrt{\mu_{1}} \sqrt{\mu_{2}}}\end{array}\right) \partial+\frac{i}{x-x_{0}}\left(\begin{array}{cc}0 & 1 \\ -1 & 0\end{array}\right)\right] B^{\mathrm{t}} A^{\mathrm{t}}$,

$H_{+}=A B\left[-I_{2} \partial^{2}+\frac{2}{\left(x-x_{0}\right)^{2}}\left(\begin{array}{ll}1 & 0 \\ 0 & 0\end{array}\right)+\lambda_{0} I_{2}\right] B^{\mathrm{t}} A^{\mathrm{t}}, \quad B=\left(\begin{array}{cc}\frac{i \sqrt{\mu_{2}}}{\sqrt{\mu_{1}-\mu_{2}}} & \frac{-\sqrt{\mu_{1}}}{\sqrt{\mu_{1}-\mu_{2}}} \\ \frac{\sqrt{\mu_{1}}}{\sqrt{\mu_{1}-\mu_{2}}} & \frac{i \sqrt{\mu_{2}}}{\sqrt{\mu_{1}-\mu_{2}}}\end{array}\right)$,

где ветвь корня $\sqrt{\mu_{1}-\mu_{2}}$ можно выбрать произвольно. Нетрудно проверить, что базис в ядре оператора симметрии $R_{\mathrm{a}}^{+}$образуют вектор-функции

$$
\begin{aligned}
& \Phi_{1}(x)=\frac{1}{x-x_{0}} A B\left(\begin{array}{l}
1 \\
0
\end{array}\right) \equiv \frac{1}{x-x_{0}} A\left(\begin{array}{c}
\frac{i \sqrt{\mu_{2}}}{\sqrt{\mu_{1}-\mu_{2}}} \\
\frac{\sqrt{\mu_{1}}}{\sqrt{\mu_{1}-\mu_{2}}}
\end{array}\right), \\
& \Phi_{2}(x)=\frac{x-x_{0}}{2} A B\left(2 i \frac{\sqrt{\mu_{1}} \sqrt{\mu_{2}}}{\mu_{1}+\mu_{2}}\right) \equiv \frac{x-x_{0}}{2} A\left(\begin{array}{c}
-i \frac{\sqrt{\mu_{2}} \sqrt{\mu_{1}-\mu_{2}}}{\mu_{1}+\mu_{2}} \\
\frac{\sqrt{\mu_{1}} \sqrt{\mu_{1}-\mu_{2}}}{\mu_{1}+\mu_{2}}
\end{array}\right),
\end{aligned}
$$


первая из которых для $H_{+}$является собственной, а вторая - формальной присоединенной:

$$
H_{+} \Phi_{1}=\lambda_{0} \Phi_{1}, \quad\left(H_{+}-\lambda_{0} I_{2}\right) \Phi_{2}=\Phi_{1} .
$$

ЧАСТНЫЙ СЛУЧАЙ $C_{3}=C_{2}^{2} \neq 0, r_{0}(x) \neq$ const. В этом случае общее решение уравнения (15) имеет вид

$$
r_{0}(x)=i \sqrt{C_{2}} \operatorname{th} \alpha\left(x-x_{0}\right), \quad \alpha=\frac{\sqrt{C_{2}}}{\sqrt{\mu_{1}} \sqrt{\mu_{2}}}
$$

где $x_{0}$ - постоянная интегрирования, выбираемая так, чтобы у функции $r_{0}(x)$ не было вещественных полюсов, и ветви корней $\sqrt{C_{2}}, \sqrt{\mu_{1}}$ и $\sqrt{\mu_{2}}$ могут выбираться произвольно. Соответственно оператор симметрии $R_{\mathrm{a}}^{+}$и гамильтониан $H_{+}$принимают вид

$$
\begin{aligned}
& R_{\mathrm{a}}^{+}=\sqrt{\mu_{1}} \sqrt{\mu_{2}} A\left[\left(\begin{array}{cc}
\frac{\sqrt{\mu_{1}}}{\sqrt{\mu_{2}}} & 0 \\
0 & \frac{\sqrt{\mu_{2}}}{\sqrt{\mu_{1}}}
\end{array}\right) \partial+i \alpha \operatorname{th}\left[\alpha\left(x-x_{0}\right)\right]\left(\begin{array}{cc}
0 & 1 \\
-1 & 0
\end{array}\right)\right] A^{\mathrm{t}}, \\
& H_{+}=A\left[-I_{2} \partial^{2}-\frac{2 \alpha^{2}}{\operatorname{ch}^{2}\left[\alpha\left(x-x_{0}\right)\right]}\left(\begin{array}{cc}
-\frac{\mu_{2}}{\mu_{1}-\mu_{2}} & i \frac{\sqrt{\mu_{1}} \sqrt{\mu_{2}}}{\mu_{1}-\mu_{2}} \\
i \frac{\sqrt{\mu_{1}} \sqrt{\mu_{2}}}{\mu_{1}-\mu_{2}} & \frac{\mu_{1}}{\mu_{1}-\mu_{2}}
\end{array}\right)+\lambda_{0} I_{2}\right] A^{\mathrm{t}}, \\
& \lambda_{0}=\frac{C_{1}+C_{2}}{\mu_{1} \mu_{2}} .
\end{aligned}
$$

Используя ту же ортогональную матрицу $B$, что и выше, оператор $H_{+}$можно привести к более простому виду:

$$
\begin{aligned}
& R_{\mathrm{a}}^{+}=\sqrt{\mu_{1}} \sqrt{\mu_{2}} A B\left[\left(\begin{array}{cc}
0 & -i \\
-i & \frac{\mu_{1}+\mu_{2}}{\sqrt{\mu_{1}} \sqrt{\mu_{2}}}
\end{array}\right) \partial+i \alpha \operatorname{th}\left[\alpha\left(x-x_{0}\right)\right]\left(\begin{array}{cc}
0 & 1 \\
-1 & 0
\end{array}\right)\right] B^{\mathrm{t}} A^{\mathrm{t}}, \\
& H_{+}=A B\left[-I_{2} \partial^{2}-\frac{2 \alpha^{2}}{\operatorname{ch}^{2}\left[\alpha\left(x-x_{0}\right)\right]}\left(\begin{array}{ll}
1 & 0 \\
0 & 0
\end{array}\right)+\lambda_{0} I_{2}\right] B^{\mathrm{t}} A^{\mathrm{t}} .
\end{aligned}
$$

Нетрудно проверить, что базис в ядре оператора симметрии $R_{\mathrm{a}}^{+}$образуют векторфункции

$$
\begin{aligned}
& \Phi_{1}(x)=\frac{1}{\operatorname{ch}\left[\alpha\left(x-x_{0}\right)\right]} A B\left(\begin{array}{l}
1 \\
0
\end{array}\right) \equiv \frac{1}{\operatorname{ch}\left[\alpha\left(x-x_{0}\right)\right]} A\left(\begin{array}{c}
\frac{i \sqrt{\mu_{2}}}{\sqrt{\mu_{1}-\mu_{2}}} \\
\frac{\sqrt{\mu_{1}}}{\sqrt{\mu_{1}-\mu_{2}}}
\end{array}\right), \\
& \Phi_{2}(x)=-\frac{\operatorname{ch}\left[\alpha\left(x-x_{0}\right)\right]}{2 \alpha^{2}} A B\left(2 i \frac{\sqrt{\mu_{1}} \sqrt{\mu_{2}}}{\mu_{1}+\mu_{2}}\right) \equiv-\frac{\operatorname{ch}\left[\alpha\left(x-x_{0}\right)\right]}{2 \alpha^{2}} A\left(\begin{array}{c}
-i \frac{\sqrt{\mu_{2}} \sqrt{\mu_{1}-\mu_{2}}}{\mu_{1}+\mu_{2}} \\
\frac{\sqrt{\mu_{1}} \sqrt{\mu_{1}-\mu_{2}}}{\mu_{1}+\mu_{2}}
\end{array}\right),
\end{aligned}
$$


первая из которых для $H_{+}$является собственной (если $\operatorname{Re} \alpha \neq 0$ ), а вторая - формальной присоединенной:

$$
H_{+} \Phi_{1}=\left(\lambda_{0}-\alpha^{2}\right) \Phi_{1}, \quad\left[H_{+}-\left(\lambda_{0}-\alpha^{2}\right) I_{2}\right] \Phi_{2}=\Phi_{1} .
$$

ЧАСТныЙ СЛУчАй $C_{2} \neq 0, C_{3}=0, r_{0}(x) \neq$ const. В этом случае общее решение уравнения (15) имеет вид

$$
r_{0}(x)=\frac{\sqrt{-2 C_{2}}}{\operatorname{ch}\left[\beta\left(x-x_{0}\right)\right]}, \quad \beta=\frac{\sqrt{-2 C_{2}}}{\sqrt{\mu_{1}} \sqrt{\mu_{2}}},
$$

где $x_{0}$ - постоянная интегрирования, выбираемая так, чтобы у функции $r_{0}(x)$ не было вещественных полюсов, и ветви корней $\sqrt{-2 C_{2}}, \sqrt{\mu_{1}}$ и $\sqrt{\mu_{2}}$ могут выбираться произвольно. Соответственно оператор симметрии $R_{\mathrm{a}}^{+}$и гамильтониан $H_{+}$принимают вид

$$
\begin{aligned}
& R_{\mathrm{a}}^{+}=\sqrt{\mu_{1}} \sqrt{\mu_{2}} A\left[\left(\begin{array}{cc}
\frac{\sqrt{\mu_{1}}}{\sqrt{\mu_{2}}} & 0 \\
0 & \frac{\sqrt{\mu_{2}}}{\sqrt{\mu_{1}}}
\end{array}\right) \partial+\frac{\beta}{\operatorname{ch}\left[\beta\left(x-x_{0}\right)\right]}\left(\begin{array}{cc}
0 & 1 \\
-1 & 0
\end{array}\right)\right] A^{\mathrm{t},} \\
& H_{+}=A\left[\begin{array}{l}
\left.-I_{2} \partial^{2}+2 \beta^{2} \frac{\sqrt{\mu_{1}} \sqrt{\mu_{2}}}{\mu_{1}-\mu_{2}}\left(\begin{array}{ll}
\frac{\sqrt{\mu_{2}} / \sqrt{\mu_{1}}}{\operatorname{ch}^{2}\left[\beta\left(x-x_{0}\right)\right]} & \frac{\operatorname{sh}\left[\beta\left(x-x_{0}\right)\right]}{\operatorname{ch}^{2}\left[\beta\left(x-x_{0}\right)\right]} \\
\frac{\operatorname{sh}\left[\beta\left(x-x_{0}\right)\right]}{\operatorname{ch}^{2}\left[\beta\left(x-x_{0}\right)\right]} & \frac{-\sqrt{\mu_{1}} / \sqrt{\mu_{2}}}{\operatorname{ch}^{2}\left[\beta\left(x-x_{0}\right)\right]}
\end{array}\right)+\left(\begin{array}{cc}
\lambda_{1} & 0 \\
0 & \lambda_{2}
\end{array}\right)\right] A^{\mathrm{t}}, \\
\lambda_{1,2}=\frac{C_{1}}{\mu_{1} \mu_{2}} \mp \frac{\beta^{2}\left(\mu_{1}+\mu_{2}\right)}{2\left(\mu_{1}-\mu_{2}\right)} \equiv \frac{1}{\mu_{1} \mu_{2}}\left[C_{1} \pm C_{2} \frac{\mu_{1}+\mu_{2}}{\mu_{1}-\mu_{2}}\right] .
\end{array}\right.
\end{aligned}
$$

Нетрудно проверить, что базис в ядре оператора симметрии $R_{\mathrm{a}}^{+}$образуют векторфункции

$$
\Phi_{1}(x)=A\left(\begin{array}{c}
\operatorname{th}\left[\beta\left(x-x_{0}\right)\right] \\
\frac{-\sqrt{\mu_{1}} / \sqrt{\mu_{2}}}{\operatorname{ch}\left[\beta\left(x-x_{0}\right)\right]}
\end{array}\right), \quad \Phi_{2}(x)=A\left(\begin{array}{c}
\frac{\sqrt{\mu_{2}} / \sqrt{\mu_{1}}}{\operatorname{ch}\left[\beta\left(x-x_{0}\right)\right]} \\
\operatorname{th}\left[\beta\left(x-x_{0}\right)\right]
\end{array}\right),
$$

являющиеся волновыми вектор-функциями $H_{+}$, соответствующими двум нижним границам непрерывного спектра $\lambda_{1}$ и $\lambda_{2}$ :

$$
H_{+} \Phi_{1}=\lambda_{1} \Phi_{1}, \quad H_{+}=\lambda_{2} \Phi_{2}
$$

ЧАСТНЫЙ СЛУчАй $C_{3} \neq C_{2}^{2}, C_{3} \neq 0, r_{0}(x) \neq$ const. В этом случае общее решение уравнения (15) можно записать в виде

$$
\int_{r_{0}\left(x_{0}\right)}^{r_{0}(x)} \frac{d r_{0}(x)}{\sqrt{r_{0}^{4}(x)+2 C_{2} r_{0}^{2}(x)+C_{3}}}= \pm \frac{i}{\sqrt{\mu_{1}} \sqrt{\mu_{2}}}\left(x-x_{0}\right),
$$

где $x_{0} \in \mathbb{R}$ и $r_{0}\left(x_{0}\right)$ - произвольные постоянные, выбираемые так, чтобы функция $r_{0}(x)$ не имела вещественных полюсов, и значения всех корней могут выбираться 
произвольно. Можно показать, что при выполнении условий

$$
\mu_{1} \mu_{2}>0, \quad C_{2} \in \mathbb{R}, \quad C_{3}<C_{2}^{2}
$$

функция $r_{0}(x)$ будет периодической. Тем самым, будут периодическими с тем же периодом коэффициент оператора $R_{\mathrm{a}}^{+}$при $\partial^{0}$ и потенциал $V_{+}(x)$ гамильтониана $H_{+}$.

ЧАСТНЫЙ СЛУЧАЙ $r_{0}(x)=$ const $\neq 0$. В этом случае общее решение системы $(12)$ имеет вид

$$
r_{0}(x)=\sqrt{\mu_{1}} \sqrt{\mu_{2}} k_{0}, \quad v_{1}(x)=\lambda_{0}, \quad v_{2}(x) \equiv v_{3}(x) \equiv 0,
$$

где $k_{0} \neq 0$ и $\lambda_{0}$ - произвольные постоянные. Соответственно оператор симметрии $R_{\mathrm{a}}^{+}$ и гамильтониан $H_{+}$принимают вид

$$
\begin{aligned}
& R_{\mathrm{a}}^{+}=\sqrt{\mu_{1}} \sqrt{\mu_{2}} A\left[\left(\begin{array}{cc}
\frac{\sqrt{\mu_{1}}}{\sqrt{\mu_{2}}} & 0 \\
0 & \frac{\sqrt{\mu_{2}}}{\sqrt{\mu_{1}}}
\end{array}\right) \partial+k_{0}\left(\begin{array}{cc}
0 & 1 \\
-1 & 0
\end{array}\right)\right] A^{\mathrm{t}}, \\
& H_{+}=A\left[-I_{2} \partial^{2}+\lambda_{0} I_{2}\right] A^{\mathrm{t}} .
\end{aligned}
$$

Нетрудно проверить, что базис в ядре оператора симметрии $R_{\mathrm{a}}^{+}$образуют векторфункции

$$
\Phi_{1}(x)=A\left(\begin{array}{c}
1 \\
-i \frac{\sqrt{\mu_{1}}}{\sqrt{\mu_{2}}}
\end{array}\right) e^{i k_{0} x}, \quad \Phi_{2}(x)=A\left(\begin{array}{c}
1 \\
i \frac{\sqrt{\mu_{1}}}{\sqrt{\mu_{2}}}
\end{array}\right) e^{-i k_{0} x},
$$

являющиеся формальными собственными вектор-функциями $H_{+}$:

$$
H_{+} \Phi_{1}=\left(\lambda_{0}+k_{0}^{2}\right) \Phi_{1}, \quad H_{+}=\left(\lambda_{0}+k_{0}^{2}\right) \Phi_{2}
$$

ЧАСТныЙ СЛУЧАй $r_{0}(x) \equiv 0$. В этом случае общее решение системы $(12)$ имеет вид

$$
r_{0}(x) \equiv 0, \quad v_{1}(x)=\frac{\lambda_{1}+\lambda_{2}}{2} \quad v_{2}(x)=\frac{\lambda_{1}-\lambda_{2}}{2}, \quad v_{3}(x) \equiv 0,
$$

где $\lambda_{1}$ и $\lambda_{2}-$ произвольные постоянные. Соответственно оператор симметрии $R_{\mathrm{a}}^{+}$ и гамильтониан $H_{+}$принимают вид

$$
R_{\mathrm{a}}^{+}=\sqrt{\mu_{1}} \sqrt{\mu_{2}} A\left[\left(\begin{array}{cc}
\frac{\sqrt{\mu_{1}}}{\sqrt{\mu_{2}}} & 0 \\
0 & \frac{\sqrt{\mu_{2}}}{\sqrt{\mu_{1}}}
\end{array}\right) \partial\right] A^{\mathrm{t}}, \quad H_{+}=A\left[-I_{2} \partial^{2}+\left(\begin{array}{cc}
\lambda_{1} & 0 \\
0 & \lambda_{2}
\end{array}\right)\right] A^{\mathrm{t}} .
$$

Нетрудно проверить, что базис в ядре оператора симметрии $R_{\mathrm{a}}^{+}$образуют векторфункции

$$
\Phi_{1}(x)=A\left(\begin{array}{l}
1 \\
0
\end{array}\right), \quad \Phi_{2}(x)=A\left(\begin{array}{l}
0 \\
1
\end{array}\right)
$$


являющиеся волновыми вектор-функциями $H_{+}$, соответствующими двум нижним границам непрерывного спектра $\lambda_{1}$ и $\lambda_{2}$ :

$$
H_{+} \Phi_{1}=\lambda_{1} \Phi_{1}, \quad H_{+}=\lambda_{2} \Phi_{2} .
$$

\subsection{2. Случай $\mu_{1}=\mu_{2} \neq 0$.}

ЧАСТный СЛУчАЙ $r_{0}(x) \not \equiv 0$. В этом случае общее решение системы $(12)$ имеет вид

$$
\begin{aligned}
r_{0}(x)=\mu_{1} k_{0}, & v_{1}(x)=\lambda_{0}, \\
v_{2}(x)=i C_{1} e^{2 i k x}-i C_{2} e^{-2 i k x}, \quad v_{3}(x) & =C_{1} e^{2 i k x}+C_{2} e^{-2 i k x},
\end{aligned}
$$

где $k_{0} \neq 0, \lambda_{0}, C_{1}$ и $C_{2}$ - произвольные постоянные. Соответственно оператор симметрии $R_{\mathrm{a}}^{+}$и гамильтониан $H_{+}$принимают вид

$$
\begin{aligned}
& R_{\mathrm{a}}^{+}=\mu_{1}\left[I_{2} \partial+\left(\begin{array}{cc}
0 & k_{0} \\
-k_{0} & 0
\end{array}\right)\right], \\
& H_{+}=-I_{2} \partial^{2}+\left(\begin{array}{cc}
i C_{1} e^{2 i k_{0} x}-i C_{2} e^{-2 i k_{0} x} & C_{1} e^{2 i k_{0} x}+C_{2} e^{-2 i k_{0} x} \\
C_{1} e^{2 i k_{0} x}+C_{2} e^{-2 i k_{0} x} & -i C_{1} e^{2 i k_{0} x}+i C_{2} e^{-2 i k_{0} x}
\end{array}\right)+\lambda_{0} I_{2} .
\end{aligned}
$$

Нетрудно проверить, что базис в ядре оператора симметрии $R_{\mathrm{a}}^{+}$образуют векторфункции

$$
\Phi_{1}(x)=\left(\begin{array}{c}
1 \\
-i
\end{array}\right) e^{i k_{0} x}, \quad \Phi_{2}(x)=\left(\begin{array}{l}
1 \\
i
\end{array}\right) e^{-i k_{0} x},
$$

из которых можно составить формальные собственные и присоединенные векторфункции $H_{+}$:

$$
\begin{gathered}
H_{+}\left[\sqrt{C_{1}} \Phi_{1} \mp i \sqrt{C_{2}} \Phi_{2}\right]=\left(\lambda_{0}+k_{0}^{2} \pm 2 \sqrt{C_{1}} \sqrt{C_{2}}\right)\left[\sqrt{C_{1}} \Phi_{1} \mp i \sqrt{C_{2}} \Phi_{2}\right], \quad C_{1} C_{2} \neq 0, \\
H_{+} \Phi_{1}=\left(\lambda_{0}+k_{0}^{2}\right) \Phi_{1}, \quad\left[H_{+}-\left(\lambda_{0}+k_{0}^{2}\right) I_{2}\right]\left(\frac{1}{2 i C_{1}} \Phi_{2}\right)=\Phi_{1}, \quad C_{1} \neq 0, \quad C_{2}=0, \\
H_{+} \Phi_{2}=\left(\lambda_{0}+k_{0}^{2}\right) \Phi_{2}, \quad\left[H_{+}-\left(\lambda_{0}+k_{0}^{2}\right) I_{2}\right]\left(\frac{-1}{2 i C_{2}} \Phi_{1}\right)=\Phi_{2}, \quad C_{1}=0, \quad C_{2} \neq 0, \\
H_{+} \Phi_{1}=\left(\lambda_{0}+k_{0}^{2}\right) \Phi_{1}, \quad H_{+} \Phi_{2}=\left(\lambda_{0}+k_{0}^{2}\right) \Phi_{2}, \quad C_{1}=C_{2}=0,
\end{gathered}
$$

где ветви корней $\sqrt{C}_{1}$ и $\sqrt{C}_{2}$ можно выбирать произвольно. Кроме того, нетрудно убедиться в том, что из $R_{\mathrm{a}}^{+}$и $H_{+}$можно составить симметричный оператор симметрии для $H_{+}$первого порядка:

$$
\frac{1}{\mu_{1}^{2}}\left(R_{\mathrm{a}}^{+}\right)^{2}+H_{+}=\left(\begin{array}{cc}
0 & 2 k_{0} \\
-2 k_{0} & 0
\end{array}\right) \partial+V_{+}(x)-k_{0}^{2} I_{2},
$$

причем

$$
\begin{aligned}
& {\left[\frac{1}{\mu_{1}^{2}}\left(R_{\mathrm{a}}^{+}\right)^{2}+H_{+}+\left(k_{0}^{2}-\lambda_{0}\right) I_{2}\right]^{2}=4\left[k_{0}^{2} H_{+}+\left(C_{1} C_{2}-k_{0}^{2} \lambda_{0}\right) I_{2}\right] \Leftrightarrow} \\
& \quad \Leftrightarrow \frac{1}{\mu_{1}^{4}}\left(R_{\mathrm{a}}^{+}\right)^{4}+\frac{2}{\mu_{1}^{2}}\left(R_{\mathrm{a}}^{+}\right)^{2}\left[H_{+}-\left(\lambda_{0}-k_{0}^{2}\right) I_{2}\right]+\left[H_{+}-\left(\lambda_{0}+k_{0}^{2}\right) I_{2}\right]^{2}=4 C_{1} C_{2} I_{2} .
\end{aligned}
$$


ЧАСТНЫЙ СЛУЧАЙ $r_{0}(x) \equiv 0$. В этом случае общее решение системы $(12)$ имеет вид

$$
r_{0}(x) \equiv 0, \quad v_{1}(x)=\lambda_{0}, \quad v_{2}(x)=C_{1}, \quad v_{3}(x)=C_{2},
$$

где $\lambda_{0}, C_{1}$ и $C_{2}-$ произвольные постоянные. Соответственно оператор симметрии $R_{\mathrm{a}}^{+}$ и гамильтониан $H_{+}$принимают вид

$$
R_{\mathrm{a}}^{+}=\mu_{1} I_{2} \partial, \quad H_{+}=-I_{2} \partial^{2}+\left(\begin{array}{cc}
C_{1} & C_{2} \\
C_{2} & -C_{1}
\end{array}\right)+\lambda_{0} I_{2}
$$

Базис в ядре оператора симметрии $R_{\mathrm{a}}^{+}$, очевидно, можно составить из постоянных вектор-функций $\Phi_{1}$ и $\Phi_{2}$, представляющих собой собственные векторы или собственный и присоединенный векторы постоянной матрицы $V_{+}$. Кроме того, из $R_{\mathrm{a}}^{+}$и $H_{+}$ можно составить симметричный оператор симметрии для $H_{+}$нулевого порядка:

$$
\frac{1}{\mu_{1}^{2}}\left(R_{\mathrm{a}}^{+}\right)^{2}+H_{+}=\left(\begin{array}{cc}
C_{1} & C_{2} \\
C_{2} & -C_{1}
\end{array}\right)+\lambda_{0} I_{2}
$$

причем

$$
\left[\frac{1}{\mu_{1}^{2}}\left(R_{\mathrm{a}}^{+}\right)^{2}+H_{+}-\lambda_{0} I_{2}\right]^{2}=\left(C_{1}^{2}+C_{2}^{2}\right) I_{2} .
$$

4.1.3. Случай $\mu_{1}=-\mu_{2} \neq 0$.

ЧАСТНЫЙ СЛУчАЙ $r_{0}(x) \not \equiv 0$. В этом случае общее решение системы $(12)$ имеет вид

$$
r_{0}(x)=\mu_{1} f(x), \quad v_{1}(x)=\lambda_{0}+f^{2}(x), \quad v_{2}(x) \equiv 0, \quad v_{3}(x)=-f^{\prime}(x),
$$

где $\lambda_{0}$ - произвольная постоянная, $f(x)$ - произвольная функция. Соответственно оператор симметрии $R_{\mathrm{a}}^{+}$и гамильтониан $H_{+}$принимают вид

$$
\begin{aligned}
& R_{\mathrm{a}}^{+}=\mu_{1} A\left[\left(\begin{array}{cc}
1 & 0 \\
0 & -1
\end{array}\right) \partial+f(x)\left(\begin{array}{cc}
0 & 1 \\
-1 & 0
\end{array}\right)\right] A^{\mathrm{t}}, \\
& H_{+}=A\left[-I_{2} \partial^{2}+\left(\begin{array}{cc}
f^{2}(x) & -f^{\prime}(x) \\
-f^{\prime}(x) & f^{2}(x)
\end{array}\right)+\lambda_{0} I_{2}\right] A^{\mathrm{t}} .
\end{aligned}
$$

Нетрудно проверить, что базис в ядре оператора симметрии $R_{\mathrm{a}}^{+}$образуют векторфункции

$$
\Phi_{1}(x)=A\left(\begin{array}{c}
1 \\
-1
\end{array}\right) \exp \left[\int_{0}^{x} f(t) d t\right], \quad \Phi_{2}(x)=A\left(\begin{array}{l}
1 \\
1
\end{array}\right) \exp \left[-\int_{0}^{x} f(t) d t\right]
$$

которые представляют собой формальные собственные вектор-функции $H_{+}$:

$$
H_{+} \Phi_{1}=\lambda_{0} \Phi_{1}, \quad H_{+} \Phi_{2}=\lambda_{0} \Phi_{2}
$$


Кроме того, нетрудно убедиться в том, что из $R_{\mathrm{a}}^{+}$и $H_{+}$можно составить симметричный оператор симметрии для $H_{+}$нулевого порядка:

$$
\frac{1}{\mu_{1}^{2}}\left(R_{\mathrm{a}}^{+}\right)^{2}+H_{+}=\lambda_{0} I_{2}
$$

ЧАСТНЫЙ СЛУЧАЙ $r_{0}(x) \equiv 0$. В этом случае общее решение системы $(12)$ имеет вид

$$
r_{0}(x) \equiv 0, \quad v_{1}(x)=\frac{\lambda_{1}+\lambda_{2}}{2}, \quad v_{2}(x)=\frac{\lambda_{1}-\lambda_{2}}{2}, \quad v_{3}(x) \equiv 0,
$$

где $\lambda_{1}$ и $\lambda_{2}$ - произвольные постоянные. Соответственно оператор симметрии $R_{\mathrm{a}}^{+}$ и гамильтониан $H_{+}$принимают вид

$$
R_{\mathrm{a}}^{+}=\mu_{1} A\left[\left(\begin{array}{cc}
1 & 0 \\
0 & -1
\end{array}\right) \partial\right] A^{\mathrm{t},} \quad H_{+}=A\left[-I_{2} \partial^{2}+\left(\begin{array}{cc}
\lambda_{1} & 0 \\
0 & \lambda_{2}
\end{array}\right)\right] A^{\mathrm{t}} .
$$

Базис в ядре оператора симметрии $R_{\mathrm{a}}^{+}$, очевидно, составляют вектор-функции

$$
\Phi_{1}=A\left(\begin{array}{l}
1 \\
0
\end{array}\right), \quad \Phi_{2}=A\left(\begin{array}{l}
0 \\
1
\end{array}\right)
$$

являющиеся волновыми вектор-функциями $H_{+}$для нижних границ непрерывного спектра $\lambda_{1}$ и $\lambda_{2}$ :

$$
H_{+} \Phi_{1}=\lambda_{1} \Phi_{1}, \quad H_{+} \Phi_{2}=\lambda_{2} \Phi_{2} .
$$

Кроме того, из $R_{\mathrm{a}}^{+}$и $H_{+}$можно составить симметричный оператор симметрии для $H_{+}$нулевого порядка:

$$
\frac{1}{\mu_{1}^{2}}\left(R_{\mathrm{a}}^{+}\right)^{2}+H_{+}=A\left(\begin{array}{cc}
\lambda_{1} & 0 \\
0 & \lambda_{2}
\end{array}\right) A^{\mathrm{t}}
$$

причем

$$
\left[\frac{1}{\mu_{1}^{2}}\left(R_{\mathrm{a}}^{+}\right)^{2}+H_{+}-\frac{\lambda_{1}+\lambda_{2}}{2} l_{2}\right]^{2}=\frac{\left(\lambda_{1}-\lambda_{2}\right)^{2}}{4} l_{2} .
$$

4.1.4. Случай $\mu_{1} \neq 0, \mu_{2}=0$.

ЧАСтный СЛУчАй $r_{0}(x) \not \equiv 0$. В этом случае общее решение системы $(12)$ имеет вид

$$
r_{0}(x)=\mu_{1} k_{0}, \quad v_{1}(x)=\lambda_{0}, \quad v_{2}(x) \equiv v_{3}(x) \equiv 0,
$$

где $\lambda_{0}, \mu_{1} \neq 0$ и $k_{0} \neq 0$ - произвольные постоянные. Соответственно оператор симметрии $R_{\mathrm{a}}^{+}$и гамильтониан $H_{+}$принимают вид

$$
R_{\mathrm{a}}^{+}=\mu_{1} A\left[\left(\begin{array}{ll}
1 & 0 \\
0 & 0
\end{array}\right) \partial+k_{0}\left(\begin{array}{cc}
0 & 1 \\
-1 & 0
\end{array}\right)\right] A^{\mathrm{t}}, \quad H_{+}=A\left[-I_{2} \partial^{2}+\lambda_{0} I_{2}\right] A^{\mathrm{t}} .
$$

Ядро $R_{\mathrm{a}}^{+}$содержит только нулевую вектор-функцию. 
ЧАСТныЙ СЛУЧАЙ $r_{0}(x) \equiv 0$. В этом случае общее решение системы $(12)$ имеет вид

$$
v_{1}(x)=\lambda_{0}+\frac{1}{2} f(x), \quad v_{2}(x)=-\frac{1}{2} f(x), \quad v_{3}(x) \equiv 0,
$$

где $\lambda_{0}$ и $f(x)$ - произвольная постоянная и произвольная функция. Соответственно оператор симметрии $R_{\mathrm{a}}^{+}$и гамильтониан $H_{+}$принимают вид

$$
R_{\mathrm{a}}^{+}=\mu_{1} A\left[\left(\begin{array}{ll}
1 & 0 \\
0 & 0
\end{array}\right) \partial\right] A^{\mathrm{t},} \quad H_{+}=A\left[-I_{2} \partial^{2}+\left(\begin{array}{cc}
0 & 0 \\
0 & f(x)
\end{array}\right)+\lambda_{0} I_{2}\right] A^{\mathrm{t}}
$$

где $\mu_{1} \neq 0$ - произвольная постоянная. Ядро $R_{\mathrm{a}}^{+}$состоит из вектор-функций вида

$$
\Phi(x)=A\left(\begin{array}{c}
C \\
\varphi(x)
\end{array}\right)
$$

где $C$ и $\varphi(x)$ - произвольная постоянная и произвольная функция. При этом $\Phi(x)$, представляющие собой формальные собственные функции $H_{+}$, имеют вид

$$
\Phi(x)=A\left(\begin{array}{l}
1 \\
0
\end{array}\right), \quad H_{+} \Phi=\lambda_{0} \Phi
$$

либо

$$
\Phi(x)=A\left(\begin{array}{c}
0 \\
\varphi(x)
\end{array}\right), \quad H_{+} \Phi=\lambda \Phi,
$$

где $\varphi(x)$ - формальная собственная функция скалярного гамильтониана

$$
-\partial^{2}+f(x)+\lambda_{0}
$$

для того же самого спектрального числа $\lambda$. Для того чтобы вектор-функция (16) была нормируемой, очевидно, необходимо и достаточно, чтобы $\varphi(x)$ была нормируемой собственной функцией гамильтониана (17).

4.1.5. Случай $\mu_{1}=\mu_{2}=0$. В этом случае общее ненулевое решение системы (12) имеет вид

$$
r_{0}(x)=r_{0}, \quad v_{1}(x)=f(x), \quad v_{2}(x) \equiv v_{3}(x) \equiv 0,
$$

где $r_{0} \neq 0$ и $f(x)$ - произвольная постоянная и произвольная функция. Соответственно оператор симметрии $R_{\mathrm{a}}^{+}$и гамильтониан $H_{+}$принимают вид

$$
R_{\mathrm{a}}^{+}=r_{0}\left(\begin{array}{cc}
0 & 1 \\
-1 & 0
\end{array}\right), \quad H_{+}=-I_{2} \partial^{2}+f(x) I_{2}
$$

и для квадрата оператора симметрии справедливо равенство

$$
\left(R_{\mathrm{a}}^{+}\right)^{2}=-r_{0}^{2} I_{2}
$$




\section{2. Антисимметричный оператор симметрии с недиагонализуемым} старшим коэффициентом. Нетрудно проверить, что антисимметричный оператор симметрии $R_{\mathrm{a}}^{+}$с недиагонализуемым коэффициентом при $\partial$ для симметричного гамильтониана $H_{+}$и сам этот гамильтониан можно представить в следующем общем виде:

$$
R_{\mathrm{a}}^{+}=\left(\begin{array}{cc}
\mu \pm \nu i & \nu \\
\nu & \mu \mp \nu i
\end{array}\right) \partial+r_{0}\left(\begin{array}{cc}
0 & 1 \\
-1 & 0
\end{array}\right), \quad H_{+}=-I_{2} \partial^{2}+\left(\begin{array}{cc}
v_{1}+v_{2} & v_{3} \\
v_{3} & v_{1}-v_{2}
\end{array}\right),
$$

где $\mu, \nu \neq 0, r_{0}=r_{0}(x), v_{1}=v_{1}(x), v_{2}=v_{2}(x)$ и $v_{3}=v_{3}(x)$ - некоторые постоянные и функции. Дальнейшее исследование проведем по отдельности для нескольких случаев и положим для простоты и без ограничения общности $\nu=1$.

4.2.1. Случай $r_{0}(x) \neq$ const. В этом случае общее решение уравнения $R_{\mathrm{a}}^{+} H_{+}=$ $H_{+} R_{\mathrm{a}}^{+}$имеет вид

$$
\begin{aligned}
R_{\mathrm{a}}^{+}= & \left(\begin{array}{cc}
\mu \pm i & 1 \\
1 & \mu \mp i
\end{array}\right) \partial \mp i \mu \alpha \operatorname{th}\left[\alpha\left(x-x_{0}\right)\right]\left(\begin{array}{cc}
0 & 1 \\
-1 & 0
\end{array}\right) \\
H_{+}= & -I_{2} \partial^{2}+\frac{\alpha^{2}}{2 \mu}\left[\gamma^{2} \operatorname{ch}^{2}\left[\alpha\left(x-x_{0}\right)\right]+\frac{\mu^{2}+1}{\operatorname{ch}^{2}\left[\alpha\left(x-x_{0}\right)\right]}\right]\left(\begin{array}{cc} 
\pm i & 1 \\
1 & \mp i
\end{array}\right)- \\
& -\frac{\alpha^{2}}{\operatorname{ch}^{2}\left[\alpha\left(x-x_{0}\right)\right]}\left(\begin{array}{cc}
1 \pm i \mu & 0 \\
0 & 1 \mp i \mu
\end{array}\right)+\lambda_{0} I_{2},
\end{aligned}
$$

где $x_{0}, \alpha \neq 0, \gamma, \lambda_{0}$ и $\mu \neq 0$ - произвольные постоянные, выбираемые так, чтобы функция $\operatorname{ch}\left[\alpha\left(x-x_{0}\right)\right]$ не имела вещественных нулей. Базис в ядре оператора симметрии $R_{\mathrm{a}}^{+}$образуют вектор-функции

$$
\Phi_{1}(x)=\operatorname{ch}\left[\alpha\left(x-x_{0}\right)\right]\left(\begin{array}{c}
1 \\
\mp i
\end{array}\right), \quad \Phi_{2}(x)=\frac{1}{\operatorname{ch}\left[\alpha\left(x-x_{0}\right)\right]}\left(\begin{array}{c}
1 \pm i \mu \\
\mp i-\mu
\end{array}\right),
$$

из которых можно составить формальные собственные и присоединенные векторфункции $H_{+}$:

$$
\begin{gathered}
H_{+}\left[\gamma \Phi_{1} \pm \Phi_{2}\right]=\left(\lambda_{0}-\alpha^{2} \mp \alpha^{2} \gamma\right)\left[\gamma \Phi_{1} \pm \Phi_{2}\right], \quad \gamma \neq 0, \\
H_{+} \Phi_{2}=\left(\lambda_{0}-\alpha^{2}\right) \Phi_{2}, \quad\left[H_{+}-\left(\lambda_{0}-\alpha^{2}\right) I_{2}\right]\left(-\frac{1}{\alpha^{2}} \Phi_{1}\right)=\Phi_{2}, \quad \gamma=0 .
\end{gathered}
$$

При этом $\Phi_{2}(x)$ в случае $\gamma=0, \operatorname{Re} \alpha \neq 0$, очевидно, является нормируемой собственной вектор-функцией $H_{+}$. Кроме того, нетрудно проверить, что выполняется равенство

$$
\left[\left(R_{\mathrm{a}}^{+}\right)^{2}+\mu^{2}\left(H_{+}-\lambda_{0} I_{2}\right)-\mu^{2} \alpha^{2} I_{2}\right]^{2}=-\mu^{4} \alpha^{2}\left[4\left(H_{+}-\lambda_{0} I_{2}\right)-\alpha^{2} \gamma^{2} I_{2}\right] .
$$

4.2.2. Случай $r_{0}(x)=$ const, $\mu \neq 0$. В этом случае общее решение уравнения $R_{\mathrm{a}}^{+} H_{+}=H_{+} R_{\mathrm{a}}^{+}$имеет вид

$R_{\mathrm{a}}^{+}=\left(\begin{array}{cc}\mu \pm i & 1 \\ 1 & \mu \mp i\end{array}\right) \partial+\mu k_{0}\left(\begin{array}{cc}0 & 1 \\ -1 & 0\end{array}\right), \quad H_{+}=-I_{2} \partial^{2}+\gamma e^{ \pm 2 i k_{0} x}\left(\begin{array}{cc} \pm i & 1 \\ 1 & \mp i\end{array}\right)+\lambda_{0} I_{2}$, 
где $\gamma, \lambda_{0}, \mu \neq 0$ и $k_{0}$ - произвольные постоянные. Базис в ядре оператора симметрии $R_{\mathrm{a}}^{+}$образуют вектор-функции

$$
\Phi_{1}(x)=e^{ \pm i k_{0} x}\left(\begin{array}{c}
1 \\
\mp i
\end{array}\right), \quad \Phi_{2}(x)=e^{\mp i k_{0} x}\left(\begin{array}{c}
1 \pm i \mu \\
\mp i-\mu
\end{array}\right)
$$

из которых можно составить формальные собственные и присоединенные векторфункции $H_{+}$:

$$
\begin{gathered}
H_{+} \Phi_{1}=\left(\lambda_{0}+k_{0}^{2}\right) \Phi_{1}, \quad H_{+} \Phi_{2}=\left(\lambda_{0}+k_{0}^{2}\right) \Phi_{2}, \quad \gamma=0, \\
H_{+} \Phi_{1}=\left(\lambda_{0}+k_{0}^{2}\right) \Phi_{1}, \quad\left[H_{+}-\left(\lambda_{0}+k_{0}^{2}\right) I_{2}\right]\left(-\frac{1}{2 \gamma \mu} \Phi_{2}\right)=\Phi_{1}, \quad \gamma \neq 0 .
\end{gathered}
$$

При этом нетрудно проверить, что

$$
\left[\left(R_{\mathrm{a}}^{+}\right)^{2}+\mu^{2}\left(H_{+}-\lambda_{0} I_{2}\right)+\mu^{2} k_{0}^{2} I_{2}\right]^{2}=4 \mu^{4} k_{0}^{2}\left(H_{+}-\lambda_{0} I_{2}\right)
$$

4.2.3. Случай $r_{0}(x)=$ const, $\mu=0$. В этом случае общее решение уравнения $R_{\mathrm{a}}^{+} H_{+}=H_{+} R_{\mathrm{a}}^{+}$имеет вид

$$
R_{\mathrm{a}}^{+}=\left(\begin{array}{cc} 
\pm i & 1 \\
1 & \mp i
\end{array}\right) \partial+r_{0}\left(\begin{array}{cc}
0 & 1 \\
-1 & 0
\end{array}\right), \quad H_{+}=-I_{2} \partial^{2}+f^{\prime}\left(\begin{array}{cc} 
\pm i & 1 \\
1 & \mp i
\end{array}\right)+\left(\lambda_{0} \pm 2 i r_{0} f\right) I_{2}
$$

где $\lambda_{0}, r_{0}$ и $f=f(x)$ - произвольные постоянные и произвольная функция. Ядро $R_{\mathrm{a}}^{+}$ при условии $r_{0} \neq 0$ содержит только нулевую вектор-функцию, а при условии $r_{0}=0$ состоит из вектор-функций вида

$$
\Phi(x)=\left(\begin{array}{c}
\varphi(x) \\
C \mp i \varphi(x)
\end{array}\right)
$$

где $C$ и $\varphi(x)$ - произвольная постоянная и произвольная функция. При этом $\Phi(x)$, представляющие собой формальные собственные функции $H_{+}$, имеют следующий вид:

$$
\begin{array}{ll}
\Phi(x)=e^{i k x}\left(\begin{array}{c}
1 \\
\mp i
\end{array}\right), & H_{+} \Phi=\left(\lambda_{0}+k^{2}\right) \Phi, \\
\Phi(x)=C\left(\begin{array}{l}
0 \\
1
\end{array}\right)+\left[a x+b+C \int_{0}^{x} f(t) d t\right]\left(\begin{array}{c}
1 \\
\mp i
\end{array}\right), & H_{+} \Phi=\lambda_{0} \Phi,
\end{array}
$$

где $a, b, C$ и $k$ - произвольные постоянные. Кроме того, нетрудно проверить, что

$$
\left(R_{\mathrm{a}}^{+}\right)^{2}=-r_{0}^{2} I_{2}
$$


4.3. Симметричный оператор симметрии. Симметричный оператор симметрии $R_{\mathrm{s}}^{+}$для симметричного гамильтониана $H_{+}$и сам этот гамильтониан можно представить в следующем общем виде:

$$
\begin{aligned}
& R_{\mathrm{s}}^{+}=\mu\left(\begin{array}{cc}
0 & 1 \\
-1 & 0
\end{array}\right) \partial+\left(\begin{array}{cc}
r_{1}(x)+r_{2}(x) & r_{3}(x) \\
r_{3}(x) & r_{1}(x)-r_{2}(x)
\end{array}\right) \\
& H_{+}=-I_{2} \partial^{2}+\left(\begin{array}{cc}
v_{1}(x)+v_{2}(x) & v_{3}(x) \\
v_{3}(x) & v_{1}(x)-v_{2}(x)
\end{array}\right)
\end{aligned}
$$

где $\mu, r_{1}(x), r_{2}(x), r_{3}(x), v_{1}(x), v_{2}(x)$ и $v_{3}(x)$ - некоторые постоянная и функции. Дальнейшее исследование проведем по отдельности для нескольких случаев.

4.3.1. Случай $\mu \neq 0$. В этом случае общее решение уравнения $R_{\mathrm{s}}^{+} H_{+}=H_{+} R_{\mathrm{s}}^{+}$ имеет вид

$$
\begin{aligned}
& R_{\mathrm{s}}^{+}=\mu\left[\left(\begin{array}{cc}
0 & 1 \\
-1 & 0
\end{array}\right) \partial+\left(\begin{array}{cc}
f_{1}(x) & f_{2}(x) \\
f_{2}(x) & -f_{1}(x)
\end{array}\right)+C I_{2}\right] \\
& H_{+}=-I_{2} \partial^{2}+\left(\begin{array}{cc}
f_{1}^{2}(x)+f_{2}^{2}(x)+f_{2}^{\prime}(x) & -f_{1}^{\prime}(x) \\
-f_{1}^{\prime}(x) & f_{1}^{2}(x)+f_{2}^{2}(x)-f_{2}^{\prime}(x)
\end{array}\right)+\lambda_{0} I_{2},
\end{aligned}
$$

где $\lambda_{0}$ и $C$ - произвольные постоянные, $f_{1}(x)$ и $f_{2}(x)$ - произвольные функции. При этом операторы $R_{\mathrm{s}}^{+}$и $H_{+}$связаны соотношением

$$
\left[\frac{1}{\mu} R_{\mathrm{s}}^{+}-C I_{2}\right]^{2}=H_{+}-\lambda_{0} I_{2}
$$

4.3.2. Случай $\mu=0$, оператор $R_{\mathrm{s}}^{+}$диагонализуем. В этом случае решение уравнения $R_{\mathrm{s}}^{+} H_{+}=H_{+} R_{\mathrm{s}}^{+}$имеет либо вид

$$
R_{\mathrm{s}}^{+}=C I_{2}, \quad H_{+}=-I_{2} \partial^{2}+\left(\begin{array}{ll}
f_{1}(x) & f_{3}(x) \\
f_{3}(x) & f_{2}(x)
\end{array}\right)
$$

где $C, f_{1}(x), f_{2}(x)$ и $f_{3}(x)$ - произвольная постоянная и произвольные функции, либо вид

$$
R_{\mathrm{s}}^{+}=A\left(\begin{array}{cc}
C_{1} & 0 \\
0 & C_{2}
\end{array}\right) A^{\mathrm{t}}, \quad H_{+}=A\left[-I_{2} \partial^{2}+\left(\begin{array}{cc}
f_{1}(x) & 0 \\
0 & f_{2}(x)
\end{array}\right)\right] A^{\mathrm{t}},
$$

где $A$ - произвольная ортогональная матрица, $C_{1}, C_{2} \neq C_{1}$ - произвольные постоянные, $f_{1}(x), f_{2}(x)$ - произвольные функции.

4.3.3. Случай $\mu=0$, оператор $R_{\mathrm{s}}^{+}$недиагонализуем. В этом случае общее решение уравнения $R_{\mathrm{s}}^{+} H_{+}=H_{+} R_{\mathrm{s}}^{+}$имеет вид

$$
R_{\mathrm{s}}^{+}=C_{1}\left(\begin{array}{cc} 
\pm i & 1 \\
1 & \mp i
\end{array}\right)+C_{2} I_{2}, \quad H_{+}=-I_{2} \partial^{2}+v_{1}(x) I_{2}+v_{3}(x)\left(\begin{array}{cc} 
\pm i & 1 \\
1 & \mp i
\end{array}\right)
$$


где $C_{1} \neq 0, C_{2}$ - произвольные постоянные, $v_{1}(x)$ и $v_{3}(x)$ - произвольные функции. Нетрудно увидеть, что ядро $R_{\mathrm{s}}^{+}$в случае $C_{2}=0$ состоит из вектор-функций вида

$$
\Phi(x)=\varphi(x)(1, \mp i)^{\mathrm{t}},
$$

где $\varphi(x)$ - произвольная функция. При этом для того чтобы $\Phi(x)$ была формальной или нормируемой собственной вектор-функцией $H_{+}$, очевидно, необходимо и достаточно, чтобы $\varphi(x)$ была соответственно формальной или нормируемой собственной функцией скалярного гамильтониана $-\partial^{2}+v_{1}(x)$ для того же спектрального числа. Кроме того, нетрудно проверить, что

$$
\left[R_{\mathrm{s}}^{+}-C_{2} I_{2}\right]^{2}=0
$$

\section{5. ЗАКЛЮЧЕНИЕ}

В заключение перечислим несколько задач для дальнейших исследований.

1. Свойства матричного оператора скрытой симметрии по аналогии с работами [8], [50]-[52].

2. Определить (не)зависимость матричных сплетающих операторов по аналогии c [8] и найти критерий зависимости, а также ответы на вопросы о наибольшем числе независимых сплетающих операторов и о базисе таких операторов.

3. Разработать методы спектрального дизайна для матричных гамильтонианов с помощью сплетающих операторов произвольного порядка и, в частности, найти критерий, которому должны удовлетворять преобразующие вектор-функции для того, чтобы получаемый с их помощью гамильтониан имел желаемые изменения в спектре по сравнению с исходным гамильтонианом. Для этого нужно обобщить на матричный случай теорему об индексах и лемму 4, приведенные в работах [12], [13].

4. Найти критерии (не)приводимости матричных сплетающих операторов и провести классификацию неприводимых и абсолютно неприводимых [41] сплетающих операторов подобно тому, как это было сделано в работах [7], [10], [11], [14], [15], [53]-[59].

\section{Список литературы}

[1] A. Lahiri, P. K. Roy, B. Bagchi, Internat. J. Modern Phys. A, 5:8 (1990), 1383-1456.

[2] F. Cooper, A. Khare, U. Sukhatme, Phys. Rep., 251:5-6 (1995), 267-385.

[3] G. Junker, Supersymmetric Methods in Quantum and Statistical Physics, Springer, Berlin, 1996.

[4] A. A. Andrianov, M. V. Ioffe, J. Phys. A, 45:50 (2012), 503001, 62 pp., arXiv: 1207.6799.

[5] А. А. Андрианов, Н. В. Борисов, М. В. Иоффе, ТМФ, 61:2 (1984), 183-198.

[6] A. A. Andrianov, M. V. Ioffe, V. P. Spiridonov, Phys. Lett. A, 174:4 (1993), 273-279, arXiv: hep-th/9303005.

[7] A. A. Andrianov, F. Cannata, J.-P. Dedonder, M. V. Ioffe, Internat. J. Modern Phys. A, 10:18 (1995), 2683-2702, arXiv: hep-th/9404061.

[8] A. A. Andrianov, A. V. Sokolov, Nucl. Phys. B, 660:1-2 (2003), 25-50, arXiv: hep-th/0301062.

[9] M. V. Ioffe, D. N. Nishnianidze, Phys. Lett. A, 327:5-6 (2004), 425-432, arXiv: hep-th/ 0404078 . 
[10] A. A. Andrianov, F. Cannata, Math. Gen., 37:43 (2004), 10297-10321, arXiv: hep-th/0407077.

[11] А. А. Андрианов, А. В. Соколов, Зап. науч. сем. ПОМИ, 335 (2006), 22-49, arXiv: 0710.5738 .

[12] A. A. Andrianov, F. Cannata, A. V. Sokolov, Nucl. Phys. B, 773:3 (2007), 107-136, arXiv: math-ph/0610024.

[13] A. V. Sokolov, Nucl. Phys. B, 773:3 (2007), 137-171, arXiv: math-ph/0610022.

[14] А. В. Соколов, Зап. науч. сем. ПОМИ, 347 (2007), 214-237, arXiv: 0903.2835.

[15] А. В. Соколов, Зап. науч. сем. ПОМИ, 374 (2010), 213-249.

[16] Ю. А. Куперин, Д. М. Латыпов, С. П. Меркурьев, ЯФ, 55:8 (1992), 2057-2060.

[17] A. A. Andrianov, M. V. Ioffe, Phys. Lett. B, 205:4 (1988), 507-510.

[18] R. D. Amado, F. Cannata, J.-P. Dedonder, Phys. Rev. A, 38:7 (1988), 3797-3800.

[19] R. D. Amado, F. Cannata, J.-P. Dedonder, Internat. J. Modern Phys. A, 5:17 (1990), 3401-3415.

[20] A. A. Andrianov, M. V. Ioffe, Phys. Lett. B, 255:4 (1991), 543-549.

[21] A. A. Andrianov, M. V. Ioffe, V.P. Spiridonov, L. Vinet, Phys. Lett. B, 272:3-4 (1991), 297-304.

[22] F. Cannata, M. V. Ioffe, Phys. Lett. B, 278:4 (1992), 399-402.

[23] F. Cannata, M. V. Ioffe, J. Phys. A: Math. Gen., 26:3 (1993), L89-L92.

[24] T. Fukui, Phys. Lett. A, 178:1-2 (1993), 1-6.

[25] L. V. Hau, J. A. Golovchenko, M. M. Burns, Phys. Rev. Lett., 75:7 (1995), 1426-1429.

[26] A. A. Andrianov, F. Cannata, D. N. Nishnianidze, M. V. Ioffe, J. Phys. A, 30:14 (1997), 5037-5050, arXiv: quant-ph/9707004.

[27] J.-M. Sparenberg, D. Baye, Phys. Rev. Lett., 79:20 (1997), 3802-3805.

[28] R. de Lima Rodrigues, P. B. da Silva Filho, A. N. Vaidya, Phys. Rev. D, 58:12 (1998), 125023, 6 pp.

[29] V. M. Goncharenko, A. P. Veselov, J. Phys. A: Math. Gen., 31:23 (1998), 5315-5326.

[30] T. K. Das, B. Chakrabarti, J. Phys. A: Math. Gen., 32:12 (1999), 2387-2394.

[31] V. M. Tkachuk, P. Roy, Phys. Lett. A, 263:4-6 (1999), 245-249, arXiv: quant-ph/9905102.

[32] A. L. Sakhnovich, J. Phys. A: Math. Gen., 36:28 (2003), 7789-7802.

[33] M. V. Ioffe, A. I. Neelov, J. Phys. A: Math. Gen., 36:10 (2003), 2493-2506, arXiv: hep-th/0302004.

[34] B. F. Samsonov, A. A. Pecheritsin, J. Phys. A: Math. Gen., 37:1 (2004), 239-250, arXiv: quant-ph/0307145.

[35] F. Cannata, M. V. Ioffe, A. I. Neelov, D. N. Nishnianidze, J. Phys. A: Math. Gen., 37:43 (2004), 10339-10348, arXiv: hep-th/0405108.

[36] M. V. Ioffe, Ş. Kuru, J. Negro, L. M. Nieto, J. Phys. A: Math. Gen., 39:22 (2006), 6987-7001, arXiv: hep-th/0603005.

[37] E. Ferraro, A. Messina, A. G. Nikitin, Phys. Rev. A, 81:4 (2010), 042108, 8 pp., arXiv: 0909.5543.

[38] A. A. Pecheritsin, A. M. Pupasov, B. F. Samsonov, J. Phys. A: Math. Theor., 44:20 (2011), 205305, 15 pp., arXiv: 1102.5255.

[39] A. G. Nikitin, Yu. Karadzhov, J. Phys. A: Math. Theor., 44:30 (2011), 305204, 21 pp.; 44:44, 445202, 24 pp., arXiv: 1107.2525.

[40] T. Tanaka, Modern Phys. Lett. A, 27:9 (2012), 1250051, 10 pp., arXiv: 1108.0480.

[41] A. V. Sokolov, Phys. Lett. A, 377:9 (2013), 655-662, arXiv: 1307.4449.

[42] A. M. Pupasov-Maksimov, Multichannel generalization of eigen-phase preserving supersymmetric transformations, arXiv: 1301.4199.

[43] F. Correa, V. Jakubský, Phys. Rev. D, 90:12 (2014), 125003, 10 pp., arXiv: 1406.2997. 
[44] T. Tanaka, Construction of quasi-solvable quantum mechanical matrix models: Lie superalgebra v.s. $\mathcal{N}$-fold Supersymmetry, arXiv: 1409.5578.

[45] D. Baye, J.-M. Sparenberg, A. M. Pupasov-Maksimov, B. F. Samsonov, J. Phys. A: Math. Theor., 47:24 (2014), 243001, 75 pp., arXiv: 1401.0439.

[46] A. V. Sokolov, J. Phys. A, 48:8 (2015), 085202, 39 pp., arXiv: 1406.0191.

[47] A. A. Andrianov, A. V. Sokolov, Phys. Lett. A, 379:4 (2015), 279-283, arXiv: 1409.6695.

[48] А. А. Андрианов, М. В. Иоффе, Д. Н. Нишнианидзе, ТМФ, 104:3 (1995), 463-478.

[49] М. А. Наймарк, Линейные дифференцалъные операторы, Наука, М., 1969.

[50] A. A. Andrianov, A. V. Sokolov, Proceedings of Institute of Mathematics of NAS of Ukraine, 50:2 (2004), 539-546.

[51] F. Correa, V. Jakubský, M. S. Plyushchay, J. Phys. A: Math. Theor., 41:48 (2008), 485303, 35 pp., arXiv: 0806.1614.

[52] A. A. Andrianov, A. V. Sokolov, SIGMA, 5 (2009), 064, 26 pp., arXiv: 0906.0549.

[53] B. F. Samsonov, Phys. Lett. A, 263:4-6 (1999), 274-280, arXiv: quant-ph/9904009.

[54] A. Khare, U. Sukhatme, J. Math. Phys., 40:11 (1999), 5473-5494, arXiv: quant-ph/9906044.

[55] D. J. Fernández C, J. Negro, L. M. Nieto, Phys. Lett. A, 275:5-6 (2000), 338-349.

[56] D. J. Fernández C, B. Mielnik, O. Rosas-Ortiz, B. F. Samsonov, J. Phys. A: Math. Gen., 35:19 (2002), 4279-4291, arXiv: quant-ph/0303051.

[57] D. J. Fernández C, R. Muñoz, A. Ramos, Phys. Lett. A, 308:1 (2003), 11-16, arXiv: quant-ph/0212026.

[58] D. J. Fernández C, E. Salinas-Hernández, J. Phys. A: Math. Gen., 36:10 (2003), 2537-2543, arXiv: quant-ph/0303123.

[59] B. F. Samsonov, Phys. Lett. A, 358:2 (2006), 105-114, arXiv: quant-ph/0602101. 